\title{
Preliminary Proposal to Use Adaptive Computer Games in the Virtual Rehabilitation Therapy
}

\author{
Mamoun Sh. Ideis, Zein Salah
}

\begin{abstract}
Virtual Rehabilitation (VR) refers to using Virtual Reality's hardware and simulations as means of exercising tools to rehabilitate patients in need. These patients will undergo their treatment exercises while playing different computer games, which helps achieve greater motivation for patients undergoing their therapeutic exercises. Virtual Rehabilitation systems adopt computer games as part of the treatment therapy. This paper present a preliminary proposal to use adaptive computer games in Virtual Rehabilitation therapy. We also present some tips in designing those adaptive computer games by use different machine learning algorithms in order to create a personalized experience for each patient, which in turn, increases the potential benefits of the treatment that each patient receives. Furthermore, we propose a method of comparing the results of treatment using the adaptive computer games with the results of using static and classical computer games.
\end{abstract}

\section{Keywords}

Virtual Rehabilitation, Physiotherapy, Adaptive Computer Games, Post-Stroke\& Game Design.

\section{INTRODUCTION}

FIFTEEN million adults are exposed to stroke worldwide every single year [1]. Patients with stroke or brain injury are subject to weakness in physical movement. The patients need to undergo physiotherapist treatment to rehabilitate the movement of their limbs [2].

Bone fractures, limb's burns or even a cut and muscular dystrophies may also affect the movement of the patient depending on the musculoskeletal injury. However, In some cases, it may also affect the cognitive and communication skills which, in turn, makes the therapy more complicated [1].

While the affected limb loses some of its functions in terms of the following three systems (physical, musculoskeletal and cognitive); the rehabilitation training aims to restore the physical movement and maintaining other skills that were affected by the stoke. This treatment must contain exercises for the affected body parts (musculoskeletal) and must also include visual tasks with cognitive, attention and memory exercises [3].

\section{BACKGROUND}

\subsection{Rehabilitation Treatment}

A rehabilitation treatment is a set of rehabilitation exercises that done by the patient with the supervision of the physiotherapist. Which required to rehabilitate the limb's movement, such as mobility strengthening exercises, walking, balanced walking programs, and normal activities of daily living [4].

According to the lack of motivation of traditional exercises in physiotherapy treatment, the computer games and virtual reality are used to motivate the patient, where the patient's motivation is a key determinant of rehabilitation outcomes [5].
Moreover, the time needed to restore the limb to the normal condition varies depending on location, the age of the patient, and the circumstances of treatment. However, longer therapeutic treatment sessions while the patient enjoys the exercises will archive greater outcomes for the treatment lifecycle [5].

\subsection{Virtual Rehabilitation}

The virtual environment is a simulation of a real world environment that generated through computer software and interact with the user to achieve sensory perception, through a human-machine interface. So, the application of using this virtual reality systems and techniques in the rehabilitation field known as Virtual Rehabilitation [6].

Virtual rehabilitation systems represent making the therapeutic exercises using virtual reality hardware and simulations in order to rehabilitate the physical, musculoskeletal, and cognitive weaknesses [3].

The advantages of using the virtual rehabilitation is the motivation of the patient as cited before [5]. Computer games make the patient enjoy his treatment sessions. Another advantage is the reduction of the need for transport the configurations, clinical settings and patient's data between patient and another or between different sessions [8].

Therefore, those virtual rehabilitation systems are in public centers and at homes. However, the economy of scale is one of the major advantages of the virtual rehabilitation, where the same devices and games can be used for different types of patients with different types of exercises.

\subsection{International Society}

The International Society for the Virtual Rehabilitation (ISVR), was established in 2009, contains a forum for engineers, clinicians, and scientist that are interested in the VR researches, where it holds the virtual rehabilitation conferences (ICVR) as one of the major activities of this society. ISVR aimed to improve the collaboration between clinicians, researchers, industry and policymakers with regard to virtual rehabilitation and telerehabilitation [7].

\section{CLASSIFICATION OF VR TOPICS}

The topics of virtual rehabilitation can be classified according to many aspects from different points of view. One of the classification methods is the use of virtual reality tools and techniques or not, and the level of immersion the system has (non-immersive, semi-immersive, or fully-immersive).

From another aspect it can be classified depending on the type of weaknesses in the patient (Physical, musculoskeletal and cognitive) or on the position of the impairment (Upper, Lower). Another way is to classify topics by the field of work and knowledge, where it can be classified into mechanical part and computer part.

\subsection{Immersion Level}

Virtual rehabilitation systems can be classified according to the 
immersion level of the virtual reality that uses in the rehabilitation process into three types which are (non, semi, full) [9].

The non-immersive level is called "desktop virtual reality", where there are no input devices and the results will be displayed only on a screen.

On the other hand, the full-immersive virtual reality the patient will do his treatment in a room or in front of huge screens that fully displays the exercise as augmented relativity world as shown in the left side of the Figure1 [9].

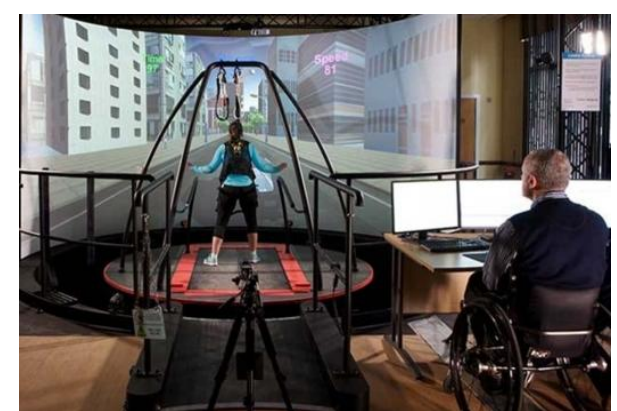

Fig. 1 Usage of Full-immersive Virtual Reality

In between, the Semi-immersive system which called "Augmented Reality" or "Hybrid System", provides a good level of the immersion without a need to huge extra devices. Gloves or eyeglasses will be used with the desktop computer [10].

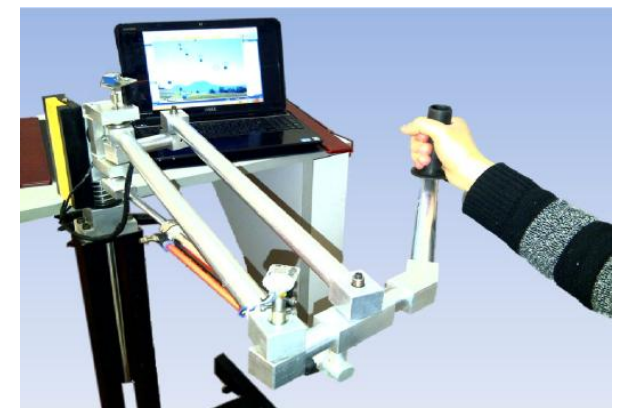

Fig. 2 Virtual rehabilitation without virtual reality

However, as shown in Figure 2, the patient interacts with the system using the computer screen and some physical sensors without using any type of virtual reality in the computer part.

Moreover, when the patient moves his limb the sensors and the controllers will calculate the limb position and understand the limb movement which in case will be transferred to the computer game which is running on the screen [2].

\subsection{Weaknesses Types}

Rehabilitation treatments and exercises differ according to the type of the weaknesses which the patient has.

Rehabilitation patients that are exposed to trauma or multiple sclerosis, cerebral palsy, or other diseases may have physical weaknesses (Motor Impairments) like the decreasing of the maximum angle of the affected limb's movement [1], [2].

So, the physiotherapist will ask the patient to make some exercise in order to extend the range of movement for the affected limb.

Moreover, in order to rehabilitate the physical part using virtual rehabilitation, the system must be designed to achieve the same value of the movement exercises by choosing the best design of the mechanical part and the best design of the computer game.
The musculoskeletal weaknesses that come from burns, cuts or other causes need to rehabilitate in another different way, which using of some weighted object in order to enhance the muscles strength by mobility strengthening exercises, for example [4].

Although, the design of the mechanical part and the computer games must take into consideration the musculoskeletal strengthening protocols.

The third type of the weaknesses is the cognitive abilities, which could be affected in more than one aspect.

Cognitive weaknesses may be in the concentration and attention skills, or in memory, or in perception process, or in other parts of the cognitive skills. The process of rehabilitating those cognitive aspects is known as "Cognitive Rehabilitation" or "Neuroplasticity" [1].

Cognitive skills improvement and recovery is one of the most design issues that must be considered in choosing or designing the computer games of the treatment exercises.

\subsection{Weaknesses Positions}

Another point of view that helps in the classification of the virtual rehabilitation topics which is classifying on the position of the affected limb on patient's body such that (upper limb part vs. lower limb part).

However, the poor sequencing and coordination of segmental movement, decreased motor impulse problems and sensory deficits are examples of problems that occur in the upper limb part of the patient's body [1].

Additionally, the ability to walk independently or walk with enough speed is one of the most important daily life activities which may be affected and needed the rehabilitation treatment as one of lower limb part weaknesses. So, some virtual rehabilitation systems (mostly the Full-immersive systems) are designed for this part.

\subsection{Mechanical Part}

When the topic of the virtual rehabilitation is classified according to the field of knowledge, it will be classified into two parts, which are the mechanical part and computer part.

The virtual rehabilitation mechanical part is composed of different parts and components connected with each other. These parts have different types and shapes with different properties. The design must provide a compromise between these properties to achieve the required shape and performance without affecting safety.

However, there are many different factors that must be considered in the mechanical part design [1] such that the machine safety, portability, cost, friction, mechanical stiffness, etc.

\subsection{Computer Part}

In order to get the motivation and enhance the rehabilitation process, an interesting computer game will be placed in front of the patient, which works in parallel with the movement of the patient's limbs to get the patient motivated to do the therapeutic exercises correctly and joyously.

Every therapeutic session consists of playing different computer games, which are designed on the bases of therapeutic protocols in order to get correct results by correct exercises. 


\section{COMPUTER GAMES DESIGN ISSUES}

The following sections consist of the design issues for choosing and designing the theoretic computer games:

\subsection{Therapeutic Exercises Based}

The major aspect of the game design for the rehabilitation process is that the computer games must be designed based on the therapeutic exercises which aims to maintain the physical part of the limb's movements.

Physiotherapeutic exercises vary from patient to another depending on the affected limb and the level of the limitation. Moreover, it depends on the age of the patient. However, the physiotherapist asks the patient to do the exercises while monitoring the evaluation of the movements by both quantitative and qualitative metrics [11]

The physical therapeutic exercises are classified into three types, which are following paths, reaching targets and objects manipulation exercises [2].

Both reaching targets and paths following exercises can be applied an empty workspace with simple movements and direct ways of sensing those movements. However, the sensing part aims to convert the limb's movement as the control inputs that decides the actions inside the software game itself.

Furthermore, manipulation games need extra objects to move and manipulate in order to achieve the goal, which needs more complicated sensing methodology.

\subsection{Categories of Games Based on Therapeutic Exercises}

Following paths games based on asking the patient to move his limb in a path or pattern of paths which focuses on the physical limb's movements and the concentration skills where that improves the eye-hand coordination.

The Steady Hand Game shown in Figure 3, is one of the examples for following paths games where the patient asked to move the end-effector of the mechanical arm in order to follow that path that drawn on the screen with the minimum number of collisions with the path line [2]. The game needs a steady hand in the physical part and needs concentration and perception skills too. Moreover, it can be improved to enhance the memory part of the cognitive skills.

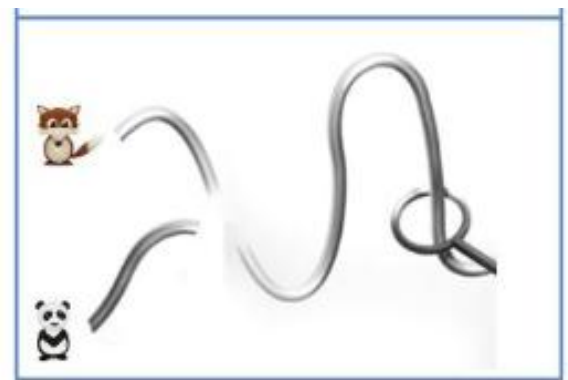

Fig. 3 Steady Hand Game

Reaching Targets Games are the most common type of computer games that can be used in the therapeutic sessions. However, most games need the player to move the controlling object to some position in order to interact with the game actions.

Pong Game is an example of the reaching targets games, where the player must move his limb in order to move the controlling object to the suitable position in order to keep the ball on the upper side without reaching the bottom of the game's world space as shown in Figure 4 [5].

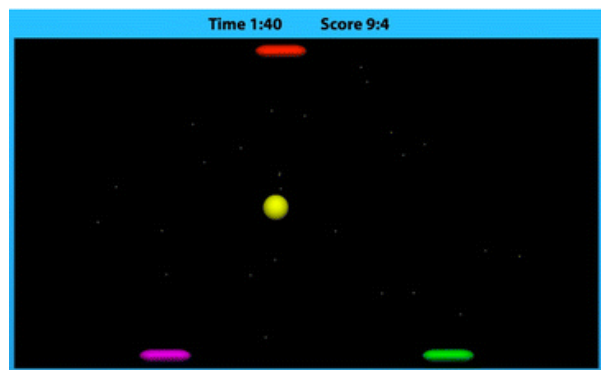

Fig. 4 Pong Game

The third category of the computer games that can be used in the therapeutic exercises and sessions which are Objects manipulation games, which based mainly on the virtual reality world and techniques.

Object manipulation ranging from mouse and keyboard as traditional input devices to use mid-air interactions in order to fetch the inputs [12]. Moreover, the result of the manipulation can be shown traditionally on the screen or it can be projected as a virtual object in suitable projection environment. Furthermore, the manipulation can be applied on both physical objects and virtual projected objects.

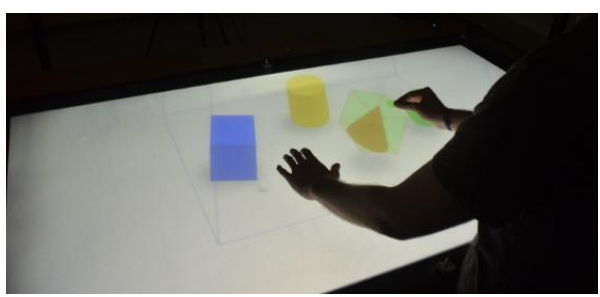

Fig. 5 Peg-in-hole Game

The Peg-in-hole game that shown in Figure 5, is an example for the games that use semi-immersive virtual reality techniques which is one of the objects manipulation games that asked the user to select, rotate and move random objects into the suitable positions in the game environment [12].

The following table that shown in Figure 6 summarized the examples of the computer games that can be used as exercises in the therapeutic treatment sessions:

\begin{tabular}{|l|l|l|}
\hline \multicolumn{1}{|c|}{ Category Name } & \multicolumn{1}{|c|}{ Example } & Reference \\
\hline Following Paths & Steady Hand Game & {$[2]$} \\
\hline Reaching Targets & Pong Game & {$[5]$} \\
\hline Objects Manipulation & Peg-in-hole Game & {$[12]$} \\
\hline
\end{tabular}

Fig. 6 Games and Categories and Examples

\subsection{Muscles Strengthening}

The virtual rehabilitation tools can be classified into four categories from the aspect of dealing with the muscular dystrophies, which are fully passive rehabilitation, active rehabilitation, active assistive rehabilitation and load-based rehabilitation.

In the fully passive rehabilitation, the patient moves his limb without any resistance and without any help from the physiotherapy. So, the rehabilitation devices also don't affect or help the patient's movement, just they reads the position of the limb and translate it into a position in the computer game, where that doesn't require extra manipulation in the game 
design side. For example, Peg-in-hole game system [12] and on of the most of rehabilitation devices that read the limb's movement from the camera are based on the passive movements.

\begin{tabular}{|l|l|}
\hline \multicolumn{1}{|c|}{ Category Name } & \multicolumn{1}{c|}{ Description } \\
\hline Fully Passive & No effect and no help from the device \\
\hline Active & Devices move the patient's limbs \\
\hline Active assistive & $\begin{array}{l}\text { Devices help the patient in his } \\
\text { movement }\end{array}$ \\
\hline Load & $\begin{array}{l}\text { Some changeable load that resist the } \\
\text { movement }\end{array}$ \\
\hline
\end{tabular}

Fig. 7 Device-Patient movement's interaction categories

As shown in the summary table in Figure 7, the last three categories affect the movement of the patient by active movement or by a resistance. So, in those cases the computer games must take in consideration the controlling part of this active movement or this resistance.

Both active categories (fully active and active assistive) require deep and special research to be applied in the computer games automatically according to the sensitivity, riskness and difficulty of controlling the active movement on an injury limb and on a patient.

In the load-based category (which can be classified as a type of passive movement in some researches), the rehabilitation device through a damper will make some resistance of the patient's movements [2]. However, the controlling of the load value can be automatic according the game design and level or according to some missions of the game, or can be manual by the therapist that monitors the patient and changes the load value manually.

A load that resists the patient's movements while making the therapeutic exercises is necessary to maintain and restore the strength of the affected muscles [1]. In this case, the patient performs an extra effort to move his limb and with different changing in loads, the patient feels the difficulty changing which is related to extra motivation [2].

\subsection{Cognitive Skills Improvement}

The knowledge of full assessment of the rehabilitation patient's cognitive strengths and weaknesses when undergoing rehabilitation will help to use the suitable methodologies to improve the patient's cognition.

As a matter of fact, the application of Computer Games in this field requires some cognitive skills in order to improve the patient's cognitive aspects.

Cognitive processes include attention, mentally focusing on some stimulus; perception, interpreting, analyzing and organizing sensory information to yield meaningful information and store them in the memory which the storage facilities and retrieval processes of cognition [3].

Actually, Cognitive abilities after a stroke or other injury could weaken in more than one aspect, these weakness may be in attention and concentration, or in memory, or in perception, or in the mental capacity, as well in other aspects like spatial awareness, for instance, neglect, the executive functioning, dysphasia and impairment of language [1].

In that case, one of the physiological theories describes the cognitive processes by: "attention, perception, and memory are identified as different cognitive activities that are involved in identifiable aspects of cognitive behavior". Those three aspects are the main direct aspects of most of the studies in this field.

On the other hand, the mental capacity, spatial awareness, executive functioning, and impairment of language can be a good area of research because of lack of search in those aspects.

Figure 8 summarize some computer games and the cognitive skills that are used in it.

\begin{tabular}{|l|l|l|}
\hline \multicolumn{1}{|c|}{ Game } & \multicolumn{1}{|c|}{ Skills } & Ref \\
\hline Steady Hand & Attention, Concentration, Memory & {$[2]$} \\
\hline Collect Money & Attention, Reaction & {$[2]$} \\
\hline Pong Game & Attention, Reaction & {$[5]$} \\
\hline Peg-in-Hole & $\begin{array}{l}\text { Concentration, Eye-hand } \\
\text { coordination }\end{array}$ & {$[12]$} \\
\hline
\end{tabular}

Fig. 8 Cognitive skills in some computer games

After all, for the purpose of using computer games in these therapeutic aspects, the game of reaching targets and collecting objects focuses on the usage of the attention and reaction cognitive skills too [5].

While the game of steady hand movements requires the usage of the attention and concentration skills in order to avoid the collisions with the bar's edges and requires some of the perception skills that are used to choose the correct path and through the whole bar to get the target. Moreover, it can be enhanced as mentioned before in order to maintain the memory skills [2].

\subsection{Motivation Aspects}

While the motivation is one of the benefits of using virtual rehabilitation technology and tools, the improvement of the motivation aspects in the game design will add some positive values to the rehabilitation treatment.

As mentioned before, cognitive skills usage in the game missions and linking the limb's movement with a movement of some mechanical resistance, both enhances the motivation of the patient and increased his satisfaction while playing the therapeutic exercises [2].

One of the motivation aspects in the design process of a computer game is the use of efficient and comfortable graphical interface design and using interactive sound actions while playing the game.

Another aspect in the game design is that the missions in the game design must be clear for the player. The player needs to understand the objective and why to accomplish it and how to do that [13]. On the other hand, the computer game must provide immediate feedback for the player about his behavior and progress moment-to-moment.

Moreover, the cooperation and competition by sharing the same game with multi players at the same time can increase the player satisfaction and motivation [5]. So, it must take into consideration in the design steps of an efficient computer game that the computer game can be played with multi players either in competition mode or in cooperation mode.

Another requirement in the game design is the need of minimizing the distraction, which can be achieved by keeping it simple and silly (K.I.S.S Principle). Therefore, the simplicity is a fundamental aspect to archive effective and motivational computer game [13]. 


\subsection{Multi-Level Games}

Furthermore, Computer games must be designed in different levels of hardness. So, multi-level computer games needed in order to achieve more motivation and more coverage for different levels of patients.

Computer games begin with a simple version of missions, tools, actions, weapons, or areas to play in and explore. After that the game over time, the new missions added and more skills needed and may some more helping tools be available too. All of that makes the game harder and requires more skills to overrun to the next levels [13].

\subsection{Progress Evaluation}

For any treatment process, there is a need to evaluate and monitor the patient's progress by both quantitative and qualitative metrics.

So, as a requirement in the game design in the virtual rehabilitation system, it must log and store every action and every progress evaluation parameter in order to notice and visualize the patient's evaluation while attending the therapeutic sessions.

Fugl-Meyer assessment (FMA) and Wolf motor function test (WMFT) are both high-level qualitative metrics assessments that evaluate each example of patient's motion as an individual where those assessments and tests need to give a mark for each listed task or movement then calculate a grade that describes the patient case [11].

Another qualitative assessment which is the Rivermead Motor Assessment (RMA) which consists of fifteen exercises for the arm movement. Therefore the physiotherapist will ask the patient to do each exercise and evaluate the movement by a grade independently [2].

On the other hand, the game results, scores, submissions points, and the progress of those quantitative metrics can be used as a good indicator for the patient's progress and evaluate the treatment level [2].

\subsection{Economy of Scale}

While the economy of scale is one of the major advantages of the virtual rehabilitation. The same tools can be used in different types of diseases and patients with different types of exercises and levels.

In order to achieve this advantage it must take into consideration in the computer game design the issue of adaptation for the game according to the patient's case. As the mechanical part of the virtual rehabilitation system which can adapt with the penitent age or affected limb, then the computer game must adapt with the patient age or the affected limb too.

Moreover, the computer game levels and difficulty must take in consideration the dynamic adjustment of the difficulty as one of the important aspects that can be adapted depending on the player skills [13]. Furthermore, this balance between game's difficulty and the patient's skills added positive values to the virtual rehabilitation economy of scale and patient's motivation too.

\section{CONCLUSION}

- There are many ways of classification for the virtual rehabilitation topics in order to get a better understanding of the whole topic.

- Rehabilitation patients affected with multi types of weaknesses in different positions and that requires the solution which covers multi aspects.

- Many issues must be considered in order to choose or design a good computer game that motivates the patient in the rehabilitation to improve the process of recovering his case.

- Carefully-selected added to the computer games design will add values to the treatment exercises and the whole treatment progress.

\section{PROPOSAL}

After discussion of the computer games and games designing tips, it can be noticed that the improvement of the games will affect positively on the treatment lifecycle.

Therefore, This study aimed to design different computer games that adapt the exercise and the game's missions and targets using some machine learning techniques based on the patient's behavior, progress and weakness points.

After designing the computer games, it is important to study the effect of adding adaptive computer games to VR treatment lifecycle by comparing the results of treatment for two groups of patients where one group uses the games in the original version and the other group with the machine learning version of the games.

\section{REFERENCES}

[1] P. Aqueveque, P. Ortega, E. Pino, F. Saavedra, E. Germany and B. Gómez, "After Stroke Movement Impairments: A Review of Current Technologies for Rehabilitation", 2017.

[2] K. A. Azzam, M. H. Takrouri, F. I. Abu-Alfielat, M. Sh. Ideis and K. A. Tahboub, "Virtual Rehabilitation RobotPreliminary Thoughts", 2013.

[3] R. N. Kalaria, R. Akinyemi, M. Ihara, "Stroke injury, cognitive impairment and vascular dementia", 2016.

[4] Janice J. Eng, "Fitness and Mobility Exercise (FAME) Program for stroke", 2012.

[5] M. Gorsic, I. Cikajlo and D. Novak, "Competitive and cooperative arm rehabilitation games played by a patient and unimpaired person: effects on motivation and exercise intensity", 2017.

[6] R. Lloréns, E. Noé, V. Naranjo, A. Borrego, J. Latorre, and M. Alcañiz, "Tracking Systems for Virtual Rehabilitation: Objective Performance vs. Subjective Experience. A Practical Scenario", 2015.

[7] http://isvr.org, 2015 Version.

[8] K. Threapleton, A. Drummond, P. Standen "Virtual rehabilitation: What are the practical barriers for homebased research?", 2016.

[9] A. S. Alqahtani, L. F. Daghestani, L. F. Ibrahim, "Environments and System Types of Virtual Reality Technology in STEM: A Survey", 2017.P. Aqueveque, P. Ortega, E. Pino, F. Saavedra, E. Germany and B. Gómez, "After Stroke Movement Impairments: A Review of Current Technologies for Rehabilitation", 2017.

[10] O. Bamodu and X. Ye, "Virtual Reality and Virtual Reality System Components", 2013.

[11] A. Vakanski,J. M. Ferguson, and S. Lee, "Metrics for Performance Evaluation of Patient Exercises during Physical Therapy", 2017. 
[12] D. F. Martins PhD Dissertation Proposal, "Manipulation of 3D Objects in Immersive Virtual Environments", 2015.
[13] C. Murphy, D. Chertoff, M. Guerrero, K. Moffitt, "DESIGN BETTER GAMES! FLOW, MOTIVATION, \& FUN", 2013. 